Revista de Matemática: Teoría y Aplicaciones 2009 16(1) : 137-147

CIMPA - UCR ISSN: 1409-2433

\title{
A MULTISTEP FUNDAMENTAL SOLUTION SCHEME FOR MODELING GROUNDWATER FLOW*
}

\author{
Juan M. Guevara-Jordan ${ }^{\dagger}$ Carmen M. Da Silva-Rodrigues ${ }^{\ddagger}$
}

Recibido/Received: 20 Feb 2008 - Aceptado/Accepted: 25 Jul 2008

\begin{abstract}
A new numerical scheme for solving transient pressure in a confined aquifer is presented. It is based on the fundamental solution method (FSM) and it combines free Green functions, superposition principle, and singular value decomposition (SVD) method to obtain an efficient computational algorithm to approximate unsteady pressure in general two dimensional groundwater problems. Its mathematical formulation avoids integral equations, is meshfree, and its new multistep approach provides very accurate approximation of full transient aquifer pressure along any period of time. The new scheme was validated with synthetic aquifers problems with constant and variable well rates. Its applications to arbitrary shaped aquifer with multiple wells is developed and analyzed. Numerical results gave evidence that the new scheme is a versatile tool and an alternative choice to boundary element methods to solve groundwater problems.
\end{abstract}

Keywords: multistep, meshless, fundamental solution method, free Green function, singular value decomposition.

\section{Resumen}

Se presenta un nuevo esquema numérico para resolver presión transitoria en un acuífero confinado. Está basado en el método de soluciones fundamentales (FSM) y combina funciones libres de Green el método de descomposición en valores singunlares (SVD) para obtener un algoritmo computacional eficiente para aproximar presión no

${ }^{*}$ This work was supported by the Consejo de Desarrollo Cientifico y Humanistico (CDCH) de la Universidad Central de Venezuela.

${ }^{\dagger}$ Escuela de Matemáticas, Universidad Central de Venezuela, Apartado 6228 Carmelitas 1010, Caracas, Venezuela. E-Mail: juan.guevara@ciencs.ucv.ve.

${ }^{\ddagger}$ Facultad de Ciencias, Universidad Central de Venezuela, Av. Los Ilustres, los Chaguaramos 1041, Caracas, Venezuela. E-Mail: carmen.dsilva@ciencs.ucv.ve. 
estática en problemas generales bidimensionales de aguas subterráneas. La formulación matemática evita el uso de ecuaciones integrales, es libre de malla, y su nuevo enfoque multipaso brinda aproximaciones muy precisas de presión transitoria en el acuífero a lo largo de cualquier periodo de tiempo. El nuevo esquema ha sido validado con problemas de acuíferos sintéticos con tasas de pozo constantes y variables. Sus aplicaciones a formas arbitrarias de acuíferos con múltiples pozos han sido desarrolladas y analizadas. Resultados numéricos han dado evidencia que el nuevo esquema es una herramienta versátil y una escogencia alternativa a los métodos de elementos de frontera para simular problemas de agua subterránea.

Palabras clave: multipaso, libre de malla, método de soluciones fundamentales, función de Green libre, descomposición en valores singulares.

Mathematics Subject Classification: 81T75, 81T80, 65C20.

\section{Introduction}

Groundwater constitutes an important component in many water systems. In particular, aquifers are one of the main sources of groundwater and their correct management is a key factor in their exploitation or conservation. In our days, numerical simulation of aquifers is a standard management tool to obtain optimum decisions or policies for these water resources $[1,2]$. Simulation of groundwater flow is based on the numerical solutions of partial differential equations. Many numerical techniques have been tested and used on these equations in this context. However, most of them can be collected in four categories. They are: finite differences, finite elements, boundary elements, and analytic methods [2]. Each one of these methods has its advantages and disadvantages, the selection of the best method is problem dependent. Consequently, the introduction of new numerical techniques is a topic of current interest. This article presents a new numerical scheme for the solution of groundwater equations for aquifers simulation. It combines the FSM, the superposition principle, and the SVD method into a single algorithm for solving transient pressure equation in groundwater modeling. The new scheme is meshless and its approximations are semianalytic, so it can be classified as an hybrid between boundary and analytic methods. Applications of FSM has been reported for elliptic and parabolic problems $[3,4]$. In the case of elliptic equations the FSM has been studied in a wide range of scientific problems with excellent results. It is wellknown that FSM has very illposed linear system, which produces numerical difficulties in its implementation. However, this problem has been eliminated with the use of SVD solvers [5] and it can be said that FSM is mature technique for solving elliptic equations. In the case of time dependent problems the FSM has been studied in the diffusion equation as a model. In general, there are two techniques for applying FSM to time dependent problems. They are the Laplace transform and finite difference discretizations in time. Both techniques reduce the time dependent problem to a finite set of elliptic equations, which are solved by the FSM. In this article a new and original multistep scheme for solving time dependent diffusion equation with the FSM is developed. It is based on the general free Green function for the transient diffusion equation and the superposition principle. Therefore, it does not rely on the solution of 
elliptic problems. The new scheme is tested in the context of groundwater problems, in which our approach has some advantages on traditional boundary methods.

The rest of this article is divided in four sections. First section presents the groundwater equations. Second section describes the FSM. Third section analyzes the tests problems and the numerical results. Finally, section four gives the conclusions and discussion.

\section{Groundwater equations}

Our work will be restricted to the modeling of groundwater flow in a confined aquifer. This type of aquifer is bounded from above and below by impervious formation. Aquifer's water is extracted through wells. Since water's level will rise above the upper confining formation inside the wells then the main variable modelled in the aquifer is its pressure. Sometimes this type of aquifer is also known as pressure aquifer. In general, the flow in any porous medium is three dimensional, but geometry of most aquifers is such that they are thin relative to their horizontal dimensions. This characteristic is usually included in the equations which neglects the vertical flow components and the water flow is essencially two dimensional. Moreover, it will be assumed that the aquifer is homogeneous and isotropic. Under all these assumptions the groundwater equation for the aquifer pressure is given by the following expression [2].

$$
\frac{\phi \mu c}{K} \frac{\partial p}{\partial t}-\left(\frac{\partial^{2} p}{\partial^{2} x}+\frac{\partial^{2} p}{\partial^{2} y}\right)=\sum_{k=1}^{n} \frac{\mu}{K h} q_{k}(t) \delta\left(x-x_{k}\right) \delta\left(y-y_{k}\right)
$$

In this equation $p$ is the aquifer's pressure, $K$ is the hydraulic conductivity or permeability, $\phi$ is porosity, $\mu$ is water viscosity, $h$ is the aquifer thickness, $q_{k}$ is the $k$ well rate, $\delta$ is the Dirac's delta function, $t$ is time and letters $x, y$ are generic coordinates of an aquifer's point. The Dirac's delta functions represent the wells positions and a singular behavior in pressure is expected near them. Sometimes equations (1) is also called pressure equation and it is of parabolic type. Consequently, its solution needs an initial condition

$$
p(x, y, 0)=p_{0}
$$

and a boundary condition

$$
\partial_{n} p(x, y)=0
$$

The initial condition is a known aquifer pressure at a reference time that we have designed by zero. In most applications $p_{0}$ is constant. The boundary condition is called Neumann's condition and it represents a sealed boundary. No flow boundary condition indicates that all water is extracted by the wells. Aquifer's pressure equation (1) plus its initial and boundary conditions is a mathematically well posed problem and it does not have a general analytic solution [6]. Therefore, the study of numerical methods for approximations of its solutions is well motivated task. 


\section{Fundamental solution method (FSM)}

This section will be subdivided in three parts. First part describes the free Green function associated to equation (1). Second part provides a brief description of a single time step of our new approach, and in the third part the new multistep scheme is explained.

\subsection{The free Green function}

In this work a free Green function is a solution of the initial value problem for the pressure equation with one well at a constant rate $q$. Under such condition the free Green function has an explicit formula

$$
G\left(\vec{x}, \overrightarrow{x_{0}}, t\right)=p_{0}+\frac{q \mu}{4 \pi K h} E i\left(\frac{c \phi \mu\left|\vec{x}-\overrightarrow{x_{0}}\right|^{2}}{4 K t}\right)
$$

where $\overrightarrow{x_{0}}$ is the well's position in the aquifer and the exponential integral function $E i(r)$ is defined by $\int_{r}^{\infty}\left(e^{-\eta} / \eta\right) d \eta$. In general, the free Green function contains an integral in time when the well's rate is not constant. This representation is more complete than equation (4), but its computational implementation is more complex and inefficient. Therefore, its use in the formulation of our multistep scheme has been avoided. The most general representation can always be approximated by a finite sum of simpler free Green functions given by (4). In this sense equation (4) is the best possible expression for computational and mathematical purposes. It is worth to mention that free Green functions are sometimes called fundamental solutions in the mathematical literature. In this work both names are synonymous.

\subsection{One step FSM}

The FSM assumes that pressure distribution can be approximated by a finite linear combination of fundamental solutions. Each fundamental solution is associated to a well. There are two types of wells in the FSM approximations: real and imaginary wells. Real wells, $\left\{\overrightarrow{x_{k}}\right\}$, are those representing the aquifer's wells and their rates, $\left\{q_{k}\right\}$, are given by field data. Imaginary wells is a set of artificial wells, $\left\{\overrightarrow{x_{j}}\right\}$,surrounding the aquifer and their rates, $\left\{Q_{i}\right\}$, must be determined by the FSM. In the case of equation (1) the FSM approximation to the pressure is given by the next expression.

$$
\begin{aligned}
p(\vec{x}, t) & =p_{0}+\sum_{k=1}^{n} \frac{q_{k} \mu}{4 \pi K h} \operatorname{Ei}\left(\frac{c \phi \mu\left|\vec{x}-\overrightarrow{x_{k}}\right|^{2}}{4 K t}\right)+ \\
& +\sum_{j=1}^{n i w} \frac{Q_{j} \mu}{4 \pi K h} \operatorname{Ei}\left(\frac{c \phi \mu\left|\vec{x}-\overrightarrow{x_{j}}\right|^{2}}{4 K t}\right)
\end{aligned}
$$

In this approximation the first term is the initial condition. The second and third terms are finite linear combinations associated to real wells and imaginary wells, respectively. The number of imaginary wells is niw. It is assumed that the fundamental solutions in the summation terms have null initial conditions. Moreover, all rates are constant, because they are frozen for one time step. In order to obtain the imaginary wells rates the FSM approximation (5) is substituted in the boundary condition (3). The resulting expression is 


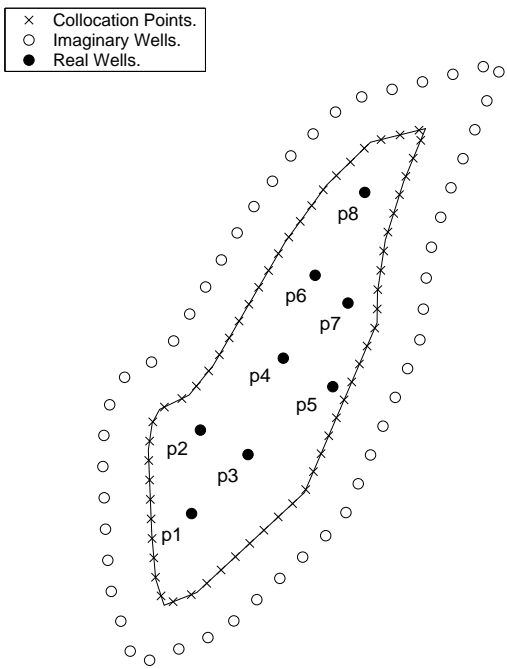

Figure 1: Well's and collocations points distributions in an irregular aquifer.

evaluated along ncp collocation points placed along the aquifer boundary. Time variable is set at the end of the time step. Each evaluation produces a linear equation for the imaginary wells rates. They are collect in a linear system

$$
A \cdot \vec{Q}=\vec{b}
$$

whose matrix $A$ has dimension $n c p \times$ niw, vector $\vec{Q}$ represents imaginary wells rates, and vector $\vec{b}$ is obtained from real wells fundamental solutions. Figure 1 shows imaginary wells, real wells, and collocation points distributions in a two dimensional irregular aquifer. Imaginary wells and collocation points are uniformly distributed. In our implementations, imaginary wells distribution follows the aquifer shape and its distance to the boundary can not be determined before hand. The number of collocation points is always greater or equal than the number of imaginary wells. This implies that linear systems (6) is overdeterminated. Very often normal equations associated to (6) are singular. This type of linear system are usually solved by QR factorizations or SVD methods [7]. In our work SVD method is used for solving (6) because it is the most robust in the FSM context [5]. SVD is applied directly to (6) and it factorizes matrix $A$ into $U \cdot S \cdot V^{T}$. Matrices $U$ and $V$ are square and orthogonal. Matrix $S$ is a rectangular matrix and its no null entries, $\{s(i, i)\}$, are ordered in a decreasing sequence along the main diagonal. These entries are the singular values of matrix $A$ and they are all no negatives. Matrix $S$ allows us to compute a pseudo inverse rectangular matrix $S^{-1}$. This pseudo inverse is a rectangular matrix whose no null entries are $1 / s(i, i)$ if $s(i, i) \neq 0$ and its remaining entries are zero. A key point in the definition of $S^{-1}$ is the criteria of a null entry in $S$. In our work null entries are those less than $10^{-6}$. Solution of (6) is given by

$$
\vec{Q}=\left(V \cdot S^{-1} \cdot U^{T}\right) \vec{b}
$$

after SVD factorization. It should be noted that $\vec{Q}$ is a least square solution, which is the best possible choice for an overdetermined system. With the imaginary well rates 
computed by (7) then all terms in the FSM approximation (5) are completely determined. Such approximation represents a single time step in our numerical scheme. In has been found that the most important parameter in the accuracy of this approximations is the number of imaginary wells and their distance to the boundary. The higher the number of imaginary wells, the better is FSM approximation. This observation is well known in the technical literature. A point of research or discussion is the optimum distance of imaginary wells to the aquifer boundary. Our tests for one time step indicates that imaginary wells should be placed at a distance less or equal to the smallest real wells distance to the boundary [8].

\subsection{Multistep FSM}

One time step scheme described in previous sections is only valid for constant rates and very short values of time $t$. These limitations are too strong and a new multistep scheme was designed to eliminate them. Without lost of generality it will be assumed that a numerical solution of groundwater pressure equation is desired on a time interval $[0, t]$ and real wells rates are time dependent. In the worst case these rates are piecewise continuous. Therefore, there exist a partition $\left\{t_{m}\right\}$ of $[0, t]$ such that for each real rate $q_{k}$ there is a piecewise constant function $\left\{q_{k}^{m}\right\}$ approximation [9]. These approximations replace the real rates in the new algorithm. This means that $q_{k} \equiv q_{k}^{m}$ on intervals $\left[t_{m-1}, t_{m}\right]$. This is not a problem because there is a finite number of real wells, so it is always possible to find a partition such that differences among approximated and real rates are negligible. The partition $\left\{t_{m}\right\}$ obtained in this way is uniform, $t_{m}-t_{m-1}=\Delta t$ is constant for all $m$, and each interval $\left[t_{m-1}, t_{m}\right]$ is a time step. In analogy to the real rates, the partition $\left\{t_{m}\right\}$ generates an associated piecewise constant rate $\left\{Q_{j}^{m}\right\}$ for each imaginary well, whose values must be computed by the FSM. The superposition principle and $E i$ properties on an uniform partitions allows us to propose the following multistep FSM approximations for the pressure determined by groundwater equations $(1),(2),(3)$ on the time interval $[0, t]$.

$$
\begin{gathered}
p\left(\vec{x}, t_{f} \equiv t\right)=p_{0}+ \\
+\frac{\mu}{4 \pi K h} \sum_{m=1}^{n t s-1} \sum_{j=1}^{n i w} Q_{j}^{m}\left(\operatorname{Ei}\left(\frac{\phi \mu c\left|\vec{x}-\overrightarrow{x_{j}}\right|^{2}}{4 K\left(t_{n t s}-t_{m-1}\right)}\right)-\operatorname{Ei}\left(\frac{\phi \mu c\left|\vec{x}-\overrightarrow{x_{j}}\right|^{2}}{4 K\left(t_{n t s}-t_{m}\right)}\right)\right)+ \\
+\frac{\mu}{4 \pi K h} \sum_{j=1}^{n i w} Q_{j}^{n t s} \operatorname{Ei}\left(\frac{\phi \mu c\left|\vec{x}-\vec{x}_{j}\right|^{2}}{4 K\left(t_{n t s}-t_{n t s-1}\right)}\right)+ \\
+\frac{\mu}{4 \pi K h} \sum_{m=1}^{n t s-1} \sum_{k=1}^{n} q_{k}^{m}\left(\operatorname{Ei}\left(\frac{\phi \mu c\left|\vec{x}-\overrightarrow{x_{k}}\right|^{2}}{4 K\left(t_{n t s}-t_{m-1}\right)}\right)-\operatorname{Ei}\left(\frac{\phi \mu c \mid}{4 K\left(t_{n t s}-t_{m}\right)}\right)\right)+ \\
+\frac{\mu}{4 \pi K h} \sum_{k=1}^{n} q_{k}^{n t s} \operatorname{Ei}\left(\frac{\phi \mu c\left|\vec{x}-\overrightarrow{x_{k}}\right|^{2}}{4 K\left(t_{n t s}-t_{n t s-1}\right)}\right)
\end{gathered}
$$

This expression contains five terms and $n t s$ denotes the number of time steps. First term contains the initial condition. Second and fourth terms represent cumulative terms which keeps track of the imaginary and real well's rates from old time steps. Fifth and fourth terms are identical to those in equation (5), they are unknown imaginary well's rates and real well's rates associated to the last time step. Expression (8) changes the number of terms in its summations for each time steps. Application of FSM to (8) produces a finite 
sequence of overdeterminated linear systems

$$
A^{(m)} \cdot \overrightarrow{Q^{(m)}}=\overrightarrow{b^{(m)}}
$$

associated to each time step. There are many possible approaches for this finite sequence of linear system. In this work the so called implicit approach is implemented. It consists in setting up a global system.

$$
\left(\begin{array}{ccccc}
A^{(1)} & 0 & \cdots & 0 & 0 \\
A^{(1)} & A^{(2)} & \cdots & 0 & 0 \\
\vdots & \vdots & \ddots & \vdots & \vdots \\
A^{(n t s-1)} & A^{(n t s-2)} & \cdots & A^{(1)} & 0 \\
A^{(n t s)} & A^{(n t s-1)} & \cdots & A^{(2)} & A^{(1)}
\end{array}\right)\left(\begin{array}{c}
\overrightarrow{Q^{(1)}} \\
\overrightarrow{Q^{(2)}} \\
\vdots \\
\overrightarrow{Q^{(n t s-1)}} \\
\overrightarrow{Q^{(n t s)}}
\end{array}\right)=\left(\begin{array}{c}
\overrightarrow{b^{(1)}} \\
\overrightarrow{b^{(2)}} \\
\vdots \\
\overrightarrow{b^{(n t s-1)}} \\
\overrightarrow{b^{(n t s)}}
\end{array}\right)
$$

and solving it by blocks with SVD. This approach has the advantage that global system's right hand side has identical expressions and its computer implementation is easier. Moreover, direct application of SVD to the global system matrix and computing a global pseudo inverse is a possible alternative, which provides correct solutions, but it is not efficient due to redundancies.

\section{Numerical results}

Our numerical results present the analysis of two test problems. They will evaluate different aspects of the new mutltistep scheme which could not be solved by the one time step FSM.

\subsection{First test problem}

This problem consist of solving pressure equation (1) in a one dimensional aquifer with one well and variable rate. The aquifer's geometry is a long two dimensional areal rectangle whose length was one hundred times its width. This configurations produces a pressure behavior similar to a one dimensional aquifer whose length is unitary and its initial pressure is two units of pressure. The well was placed at a distance equal 0.001 from the left boundary. Its rate was given by the piecewise constant function

$$
q(t)=H(t)+(0.003-1) H(t-1 / 3)+(1.4-0.003) H(t-2 / 3)
$$

where $H(t)$ is a step function equal to one if $t>0$ and zero elsewhere. Aquifer's pressure was computed with the FSM on the time interval $[0,1]$. Analytic solution for this test problem was calculated by separation of variables. This solution was used to evaluate the accuracy of the numerical approximations obtained by FSM. Imaginary wells were placed at outside the aquifer, they have distance of 0.001 to their nearest aquifer's side. This distance was determined by reflection of the real well to its nearest boundary. One special characteristic of this test problem is that its number of imaginary wells is not a 


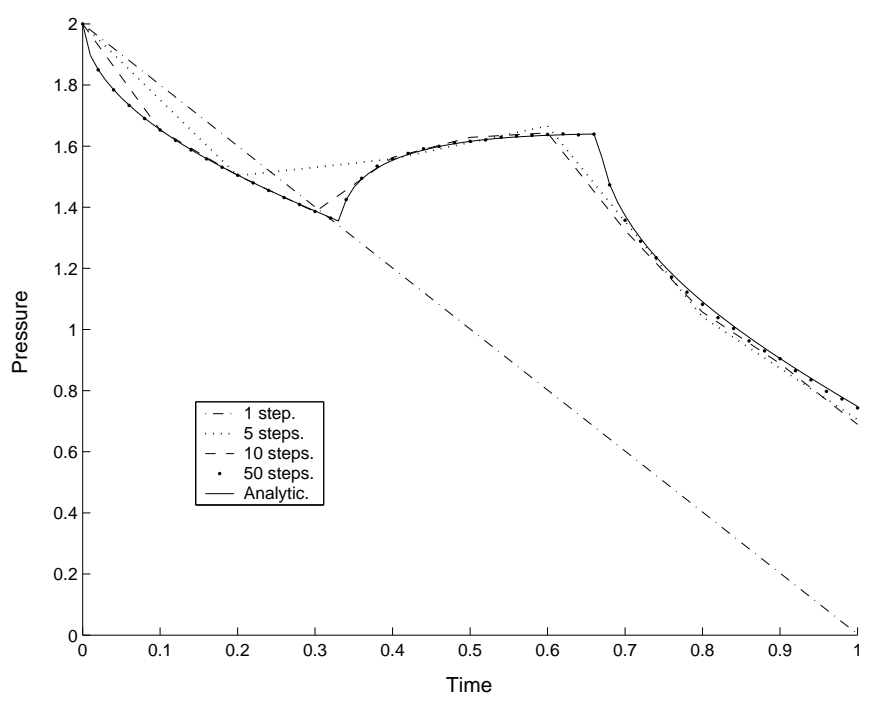

Figure 2: Analytic and numerical pressures near the well.

variable. Only two imaginary wells are possible. Therefore, numerical solution behavior is only function of the number of times steps in our new multistep scheme. Figure 2 represents a set four different pressure solutions computed with the mutistep scheme FSM for different number of time steps. Pressures are measure at a very close point to the well and their behavior are showed as function of time. Analytic solution is displayed in a strong black line. Its shape has the correct physical behavior associated with the variable rate $q(t)$. At initial times there is a constant rate of 1 and pressure decreases its value with time. As time reaches the value $1 / 3$ the rate changes to 0.003 , so pressure stars to increase its value. This increment in pressure is expected because there is almost no withdraw of water from the aquifer. Next, at time $2 / 3$ the well increases it rate to 1.4 , so the pressure decreases its value due to withdraw of water. Pressure compute with a single time step of size 1 is unable to approximate analytic solution. In fact this numerical solution is completely wrong for all time. Numerical pressure computed with five time steps of size $1 / 5$ shows some of the behaviors in the analytic solution. However, it missed the depression between at time $1 / 3$. Next numerical pressure was obtained with ten times steps of size 1/10 and it reproduces the three behaviors of the analytic solution, but it is not enough accurate. Finally a numerical pressure computed with fifty time steps provides enough resolution to match the analytic pressure along the time interval. Comparison of numerical and analytic pressures provides a strong evidence of the convergence of the new scheme. Moreover, it shows that the new multistep scheme is a necessary extension for dealing with time dependent problems.

\subsection{Second test problem}

This test consists of determining the drainage are of each well in a two dimensional irregular reservoirs as described in figure 1. Aquifer properties are showed table 1. 
Table 1: Aquifer's properties

\begin{tabular}{l|l}
\hline \hline Property & Value \\
\hline Area & $578160 \mathrm{ft}^{2}$ \\
Thickness & $150 \mathrm{ft}$ \\
Porosity & $23 \mathrm{percent}$ \\
Viscosity & $0.72 \mathrm{cp}$ \\
Compressibility & $10^{-5} 1 / \mathrm{psi}$ \\
Permeability & $100 \mathrm{mDarcys}$ \\
Initial pressure & $3000 \mathrm{psi}$ \\
\hline \hline
\end{tabular}

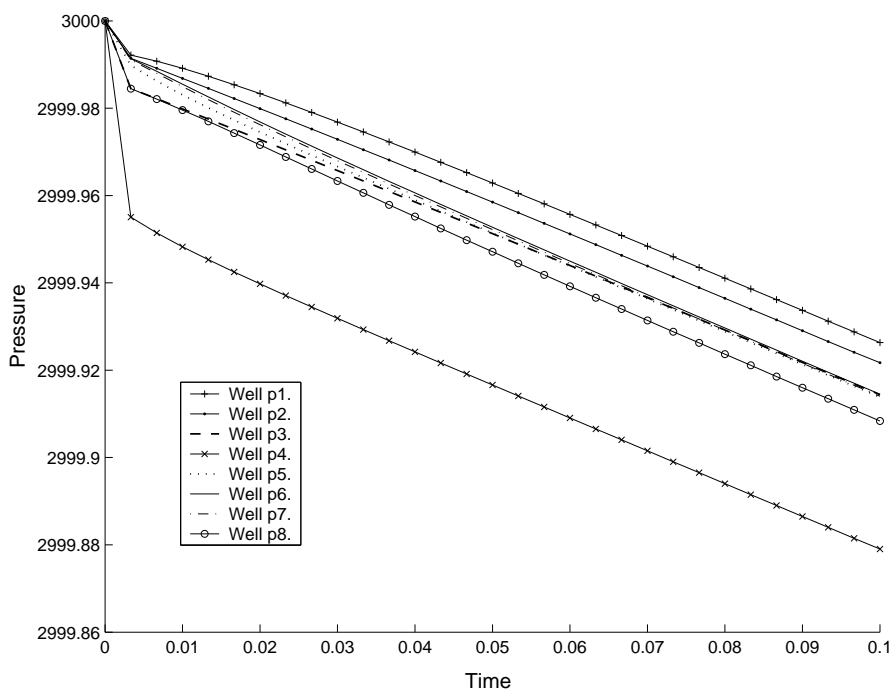

Figure 3: Pressure head evolution at wells as a function of time.

Imaginary wells were placed $200 \mathrm{ft}$ outside of the aquifer, this value was found by tuning. It is assumed that all wells in the aquifer are extracting water. Their constant rates are $q_{1}=q_{2}=q_{6}=q_{7}=100 \mathrm{stb} /$ day, $q_{3}=q_{6}=200 \mathrm{stb} / \mathrm{d}$, and $q_{4}=600 \mathrm{stb} / \mathrm{d}$. The new multistep scheme for FSM was used to compute the pressure distribution as a function of time. This solution was on an interval of time long enough to obtain an steady state pressure in the aquifer. The steady state condition is observed at the wells because their pressure head as function of time is an inclined straight line. Figure 3 shows the numerical pressure heads for each well. After time $t=0.07 \mathrm{~d}$ all these pressures have a linear behaviors, which means that a steady state condition has been reached in the aquifer. Under steady state condition the Laplacian in equation (1) is zero and the following relations results around each well.

$$
\frac{\partial p}{\partial t}=-\frac{q_{k}}{c A_{k} h \phi}
$$

In this equation $A_{k}$ is the drainage area associated to the $k$ real well and pressure derivative 


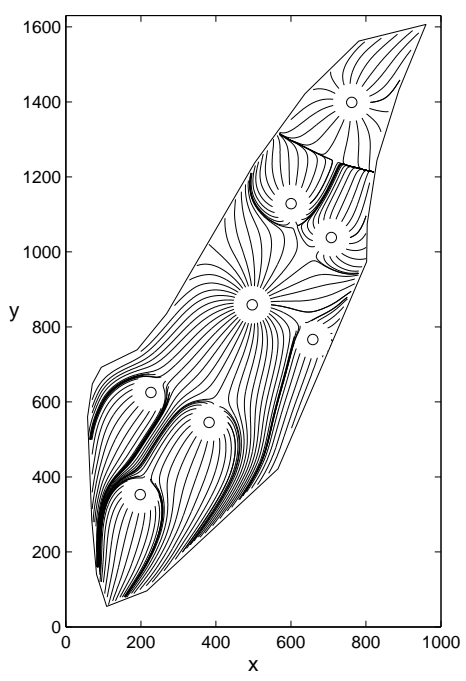

Figure 4: Streamline distribution obtained from steady state FSM pressure approximation.

is the straight line slope in the pressure head. Since all the other variables in that equation are known then it is possible to obtain an exact value for the drainage area for each well. These areas were computed with the pressure slopes from figure 3 . The pressure slope was -0.802690 for all wells. It was found that drainage areas for wells $p_{1}, p_{2}, p_{5}, p_{6}, p_{7}$ was $36110.42 f t^{2}$; for wells $p_{3}, p_{8}$ was $72215.22 f t^{2}$; and for well $p_{4}$ was $216638.94 \mathrm{ft}^{2}$. There is not a simple form to validate this results because no analytic solution exist for it. However, an analysis of the drainage areas computed shows that well $p_{4}$ drains an area six time bigger than those drained by wells $p_{1}, p_{2}, p_{5}, p_{6}, p_{7}$ and three times bigger than the area of wells $p_{3}, p_{8}$. This observation was expected because same relation is observe in the real wells rates. Moreover, addition of drained areas is equal to the aquifer's area. Therefore, the drainage areas obtained from the pressure slopes computed with the multistep scheme are physical correct and consistent. In order to appreciate the global behavior of the pressure and ratify the correct value and proportions of drainage areas, then an streamline distribution of the aquifer was obtained from the steady state FSM's pressure approximation. Streamline distributions is represented in figure 4. It provides a global and qualitative evidence that drainage areas obtained by the well head pressure slopes are correct. Consequently, it may be concluded that pressure approximations obtained from the new multistep scheme for FSM are correct.

\section{Conclusions and discussion}

A new mutlistep scheme for FSM was presented and developed for the pressure equation in groundwater modeling. The new scheme is based on diffusion equation fundamental solution and it does not need auxiliary elliptic equations in its formulation. Since the scheme is truly meshfree or meshless then it needs to keep track of all it rates values from previous time steps. Linear system generated by the scheme were analyzed and two 
possible computational strategies for its solution were elaborated. The scheme was tested in two different problems. One of them gave strong evidence that the new scheme is able to solve transient problems with variable rate. Second test problem evaluated the capability of the scheme of computing reliable pressure at long times when aquifer reaches steady state conditions. An important area of research, in which there is not a general theory, is the optimum position of imaginary wells around the aquifer. Also extensions of the scheme to heterogenous problems is necessary as well as its convergence analysis.

\section{References}

[1] Kresic, N. (2007) Hydrogeology and Groundwater Modeling. CRC Press, Boca Raton.

[2] Bear, J.; Verruijt, A. (1987) Modeling Groundwater Flow and Pollution. Reidel Publishing, Dordrecht.

[3] Fairweather, G.; Karageorghis, A.(1998) "The method of fundamental solutions for elliptic boundary value problems", Advances in Computational Mathematics, 9: 69ss.

[4] Golberg, M.A. ; Chen, C.S. (1998) "The method of fundamental solutions for potential, Helmholtz, and diffusion problems", Boundary Integral Methods-Numerical Aspecs, M.A. Golberg (Ed.), Computational Mechanics Publicaction, Southamptom: $103 \mathrm{ss}$.

[5] Ramachandran, P.A. (2001) "Method of fundamental solutions: singular value decomposition analysis", Communications in Numerical Methods in Enginnering, 18: 789 ss.

[6] DuChateau, P.; Zachmann, D. (1989) Applied Partial Differential Equations. Dover Publishing, Mineola.

[7] Golub, G.H.; Van Loan, C.F. (1996) Matrix Computations. Johns Hopkins University Press, Baltimore.

[8] Guevara-Jordan, J.M.; Castillo, M.; Villalta, D. (2006) "A meshfree method for the pressure equation in oil field problems", In: B. Gamez, D. Ojeda, M. Cerrolaza (Eds.), Simulación y Modelaje en Ingenieria y Ciencias, Editorial Sociedad Venezolana de Métodos Numéricos en Ingeniería, Venezuela, pp. TM 21.

[9] Rudin, W. (1976) Principles of Mathematical Analysis. McGraw-Hill Book Company, New York. 